\title{
Connections between Certain Subclasses of Analytic Univalent Functions Based on Operators
}

\author{
R. Ezhilarasi, ${ }^{1}$ T. V. Sudharsan, ${ }^{1}$ Maisarah Haji Mohd, ${ }^{2}$ and K. G. Subramanian ${ }^{3}$ \\ ${ }^{1}$ Department of Mathematics, SIVET College, Chennai 600 073, India \\ ${ }^{2}$ School of Mathematical Sciences, Universiti Sains Malaysia, 11800 Penang, Malaysia \\ ${ }^{3}$ Department of Mathematics, Madras Christian College, Chennai 600 059, India
}

Correspondence should be addressed to K. G. Subramanian; kgsmani1948@gmail.com

Received 19 August 2017; Accepted 17 October 2017; Published 15 November 2017

Academic Editor: Arcadii Z. Grinshpan

Copyright (C) 2017 R. Ezhilarasi et al. This is an open access article distributed under the Creative Commons Attribution License, which permits unrestricted use, distribution, and reproduction in any medium, provided the original work is properly cited.

In this paper, by applying the Hohlov linear operator, connections between the class $\operatorname{SD}(\alpha), \alpha \geq 0$, and two subclasses of the class $A$ of normalized analytic functions are established. Also an integral operator related to hypergeometric function is considered.

\section{Introduction}

Let $A$ denote the family of functions $f$ that are analytic in the open unit disk $\Delta=\{z:|z|<1\}$ with the normalization

$$
f(z)=z+\sum_{n=2}^{\infty} a_{n} z^{n} .
$$

Let $S$ denote the subclass of functions in $A$ which are also univalent in $\triangle$. A well-known subclass of $S$ is the class ST (see, e.g., [1]) of starlike functions $f$ of the form (1), satisfying $\operatorname{Re}\left\{z f^{\prime}(z) / f(z)\right\}>0, z \in \Delta$. Another class $\operatorname{UCD}(\alpha), \alpha \geq 0$, introduced in [2], consists of functions $f \in A$ satisfying $\operatorname{Re}\left(f^{\prime}(z)\right) \geq \alpha\left|z f^{\prime \prime}(z)\right|, z \in \Delta$. Various properties of this class have been obtained in [2-4]. A related class $\operatorname{SD}(\alpha)$ has been recently considered in [5], initially introduced in [6]. A function $f$ of the form (1) is said to be in the class $\operatorname{SD}(\alpha)$ if

$$
\operatorname{Re}\left\{\frac{f(z)}{z}\right\} \geq \alpha\left|f^{\prime}(z)-\frac{f(z)}{z}\right|, \quad \text { for } \alpha \geq 0 \text {. }
$$

Theorem 1 (see [5]). A function $f$ of the form (1) is in the class $S D(\alpha)$ if

$$
\sum_{n=2}^{\infty}[1+\alpha(n-1)]\left|a_{n}\right| \leq 1 .
$$

Ponnusamy and Ronning [7] introduced and studied the class $R_{\eta}(\beta) \subset S,(0 \leq \beta<1)$ of functions $f \in A$ for which there exists a number $\eta \in(-\pi / 2, \pi / 2)$ such that $\operatorname{Re}\left\{e^{i \eta}\left[f^{\prime}(z)-\beta\right]\right\}>$ $0, z \in \triangle$. If the function $f$ of the form (1) belongs to the class $R_{\eta}(\beta)$, then

$$
\left|a_{n}\right| \leq \frac{2(1-\beta) \cos \eta}{n}, \quad(n \in N \backslash\{1\}) .
$$

For complex numbers $a, b$, and $c(c \neq 0,-1,-2, \ldots)$, the Gaussian hypergeometric function ${ }_{2} F_{1}(z)$ is defined by

$$
{ }_{2} F_{1}(z)={ }_{2} F_{1}(a, b, c ; z)=\sum_{n=0}^{\infty} \frac{(a)_{n}(b)_{n}}{(c)_{n}} \frac{z^{n}}{n !},
$$

where $(\lambda)_{n}$ is the Pochhammer symbol given by

$$
\begin{aligned}
(\lambda)_{n} & = \begin{cases}1, & n=0 \\
\lambda(\lambda+1)(\lambda+2) \cdots(\lambda+n-1), & n \in N\end{cases} \\
& =\frac{\Gamma(\lambda+n)}{\Gamma(\lambda)} .
\end{aligned}
$$

It is known that

$$
\begin{aligned}
{ }_{2} F_{1}(a, b, c ; 1)= & \frac{\Gamma(c) \Gamma(c-a-b)}{\Gamma(c-a) \Gamma(c-b)}, \\
& \quad \operatorname{Re}(c-a-b)>0, c \neq 0,-1,-2, \ldots .
\end{aligned}
$$


The Hadamard product (or convolution) of two functions $f$ defined by (1) and $g$ given by $g(z)=z+\sum_{n=2}^{\infty} b_{n} z^{n}$ is defined by

$$
(f * g)(z)=z+\sum_{n=2}^{\infty} a_{n} b_{n} z^{n}
$$

Hohlov [8] introduced a linear operator $I_{a, b, c}: A \rightarrow A$, corresponding to the Gaussian hypergeometric function ${ }_{2} F_{1}$ which is defined by the convolution

$$
\left[I_{a, b, c}(f)\right](z)=z_{2} F_{1}(a, b, c, z) * f(z), \quad f \in A .
$$

For a function $f$ of the form (1), we have

$$
\left(I_{a, b, c}(f)\right)(z)=z+\sum_{n=2}^{\infty} \frac{(a)_{n-1}(b)_{n-1}}{(c)_{n-1}(1)_{n-1}} a_{n} z^{n}, \quad z \in \triangle .
$$

The operator $I_{a, b, c}$ is a natural extension of several operators such as Alexander, Libera, Bernardi, and Carlson-Shaffer operators denoted, respectively, by $\mathscr{A}, \mathfrak{L}, \mathscr{B}$, and $\mathfrak{Q}(a, c)$.

Motivated by the work of Thulasiram et al. [9], in this paper, by applying the linear operator $I_{a, b, c}$, we establish some interesting connections between the class $\mathrm{SD}(\alpha)$ and the classes ST and $R_{\eta}(\beta),(\beta<1)$ consisting of functions $f$ given by (1). Also we consider an integral operator related to the hypergeometric functions.

\section{Main Results}

In the sequel the function $f \in A$ is given by (1).

Theorem 2. Let $a, b \in C \backslash\{0\}$. Also let $c$ be a real number such that $c>|a|+|b|+2$. If $f \in S T$ and if the inequality

$$
\begin{aligned}
& \frac{\Gamma(c) \Gamma(c-|a|-|b|)}{\Gamma(c-|a|) \Gamma(c-|b|)}\left[\frac { | a b | } { ( c - | a | - | b | - 1 ) } \left(\frac{\alpha(|a|+1)(|b|+1)}{(c-|a|-|b|-2)}\right.\right. \\
& \quad+2 \alpha+1)+1] \leq 2
\end{aligned}
$$

is satisfied, then $I_{a, b, c}(f) \in S D(\alpha)$.

Proof. Let $f \in \mathrm{ST}$. Applying the well-known estimate due to Nevanlinna [10] for the coefficients of the functions $f \in \mathrm{ST}$, in view of Theorem 1 , we need to prove that

$$
\sum_{n=2}^{\infty} n(1+\alpha(n-1))\left|\frac{(a)_{n-1}(b)_{n-1}}{(c)_{n-1}(1)_{n-1}}\right| \leq 1 \text {. }
$$

By virtue of the relation $\left|(d)_{n}\right| \leq(|d|)_{n}$, and on writing $n+2=$ $(n+1)+1$ and $(n+2)^{2}=(n+1)^{2}+2(n+1)+1$ and using the fact that $(a)_{n+k}=(a)_{k}(a+k)_{n}$, we have

$$
\begin{aligned}
& \sum_{n=2}^{\infty} n(1+\alpha(n-1))\left|\frac{(a)_{n-1}(b)_{n-1}}{(c)_{n-1}(1)_{n-1}}\right| \leq \sum_{n=2}^{\infty} n(1+\alpha(n-1)) \frac{(|a|)_{n-1}(|b|)_{n-1}}{(c)_{n-1}(1)_{n-1}} \\
& =\sum_{n=1}^{\infty}\left\{(n+1)+\alpha\left[(n+1)^{2}-(n+1)\right]\right\} \frac{(|a|)_{n}(|b|)_{n}}{(c)_{n}(1)_{n}} \\
& =\alpha \sum_{n=0}^{\infty}(n+2)^{2} \frac{(|a|)_{n+1}(|b|)_{n+1}}{(c)_{n+1}(1)_{n+1}}+(1-\alpha) \sum_{n=0}^{\infty}(n+2) \frac{(|a|)_{n+1}(|b|)_{n+1}}{(c)_{n+1}(1)_{n+1}} \\
& =\alpha \sum_{n=0}^{\infty} \frac{(n+1)^{2}(|a|)_{n+1}(|b|)_{n+1}}{(c)_{n+1}(1)_{n+1}}+2 \alpha \sum_{n=0}^{\infty} \frac{(n+1)(|a|)_{n+1}(|b|)_{n+1}}{(c)_{n+1}(1)_{n+1}} \\
& +(1-\alpha) \sum_{n=0}^{\infty}(n+1) \frac{(|a|)_{n+1}(|b|)_{n+1}}{(c)_{n+1}(1)_{n+1}}+\sum_{n=0}^{\infty} \frac{(|a|)_{n+1}(|b|)_{n+1}}{(c)_{n+1}(1)_{n+1}} \\
& =\alpha \sum_{n=0}^{\infty} \frac{(|a|)_{n+2}(|b|)_{n+2}}{(c)_{n+2}(1)_{n}}+[2 \alpha+1] \sum_{n=0}^{\infty} \frac{(|a|)_{n+1}(|b|)_{n+1}}{(c)_{n+1}(1)_{n}}+\sum_{n=0}^{\infty} \frac{(|a|)_{n}(|b|)_{n}}{(c)_{n}(1)_{n}}-1 \\
& =\frac{\alpha(|a|)_{2}(|b|)_{2}}{(c)_{2}} \sum_{n=0}^{\infty} \frac{(|a|+2)_{n}(|b|+2)_{n}}{(c+2)_{n}(1)_{n}}+(2 \alpha+1) \frac{|a b|}{c} \sum_{n=0}^{\infty} \frac{(|a|+1)_{n}(|b|+1)_{n}}{(c+1)_{n}(1)_{n}} \\
& +\sum_{n=0}^{\infty} \frac{(|a|)_{n}(|b|)_{n}}{(c)_{n}(1)_{n}}-1 \\
& =\frac{\alpha(|a|)_{2}(|b|)_{2}}{(c)_{2}} \frac{\Gamma(c+2) \Gamma(c-|a|-|b|-2)}{\Gamma(c-|a|) \Gamma(c-|b|)}
\end{aligned}
$$




$$
\begin{aligned}
& +(2 \alpha+1) \frac{|a b|}{c} \frac{\Gamma(c+1) \Gamma(c-|a|-|b|-1)}{\Gamma(c-|a|) \Gamma(c-|b|)}+\frac{\Gamma(c) \Gamma(c-|a|-|b|)}{\Gamma(c-|a|) \Gamma(c-|b|)}-1 \\
= & \frac{\Gamma(c) \Gamma(c-|a|-|b|)}{\Gamma(c-|a|) \Gamma(c-|b|)}\left\{\frac{|a b|}{(c-|a|-|b|-1)}\left[\frac{\alpha(|a|+1)(|b|+1)}{(c-|a|-|b|-2)}+2 \alpha+1\right]+1\right\}-1 \\
\leq & 1,
\end{aligned}
$$

which is satisfied by the hypothesis.

On setting $b=\bar{a}$, an improvement of the assertion of Theorem 2 is obtained as given in Theorem 3 .

Theorem 3. Let $a \in C \backslash\{0\}$. Also let $c$ be a real number such that $c>\max \{0,2 \operatorname{Re}(a)+2\}$. If $f \in S T$, and if the inequality

$$
\begin{aligned}
& \frac{\Gamma(c) \Gamma(c-2 \operatorname{Re}(a))}{\Gamma(c-a) \Gamma(c-\bar{a})}\left\{\frac { | a | ^ { 2 } } { ( c - 2 \operatorname { R e } ( a ) - 1 ) } \left[\frac{\alpha(a+1)(\bar{a}+1)}{c-2 \operatorname{Re}(a)-2}\right.\right. \\
& +2 \alpha+1]+1\} \leq 2
\end{aligned}
$$

is satisfied, then $I_{a, \bar{a}, c}(f) \in S D(\alpha)$.

Theorem 4. Let $a, b \in C \backslash\{0\}$. Also, let c be a real number such that $c>|a|+|b|,|a| \neq 1,|b| \neq 1$. If $f \in R_{\eta}(\beta),(\beta<1)$ and the inequality

$$
\begin{aligned}
& \frac{2(1-\beta) \cos \eta}{(|a|-1)(|b|-1)}\left[\frac{\Gamma(c) \Gamma(c-|a|-|b|)}{\Gamma(c-|a|) \Gamma(c-|b|)}[c-|a|-|b|\right. \\
& +\alpha(|a b|+1-c)]-[c+|a b|-|a|-|b| \\
& -\alpha(c-1)]] \leq 1
\end{aligned}
$$

is satisfied, then $I_{a, b, c}(f) \in S D(\alpha)$.

Proof. Let $f$ be of the form (1) and let $f \in R_{\eta}(\beta),(\beta<1)$. By virtue of Theorem 1 and in view of (10), it remains to show that

$$
\sum_{n=2}^{\infty}(1+\alpha(n-1))\left|\frac{(a)_{n-1}(b)_{n-1}}{(c)_{n-1}(1)_{n-1}} a_{n}\right| \leq 1 .
$$

Using the inequality (4) and the relations $(d)_{n}=d(d+1)_{n-1}$ and $\left|(d)_{n}\right| \leq(|d|)_{n}$, we obtain that

$$
\begin{aligned}
& \sum_{n=2}^{\infty}(1+\alpha(n-1))\left|\frac{(a)_{n-1}(b)_{n-1}}{(c)_{n-1}(1)_{n-1}} a_{n}\right| \leq 2(1-\beta) \\
& \cdot \cos \eta \sum_{n=2}^{\infty} \frac{(1+\alpha(n-1))}{n} \frac{(|a|)_{n-1}(|b|)_{n-1}}{(c)_{n-1}(1)_{n-1}}=2 \alpha(1-\beta) \\
& \cdot \cos \eta \sum_{n=2}^{\infty} \frac{(|a|)_{n-1}(|b|)_{n-1}}{(c)_{n-1}(1)_{n-1}}+2(1-\alpha)(1-\beta) \\
& \cdot \cos \eta \sum_{n=2}^{\infty} \frac{(|a|)_{n-1}(|b|)_{n-1}}{(c)_{n-1}(1)_{n}}=2 \alpha(1-\beta) \cos \eta \sum_{n=1}^{\infty} \frac{(|a|)_{n}(|b|)_{n}}{(c)_{n}(1)_{n}}
\end{aligned}
$$

$$
\begin{aligned}
& +2(1-\alpha)(1-\beta) \\
& \cdot \cos \eta \sum_{n=2}^{\infty} \frac{\left((|a|-1)_{n} /(|a|-1)\right)\left((|b|-1)_{n} /(|b|-1)\right)}{\left((c-1)_{n} /(c-1)\right)(1)_{n}}=2 \alpha(1 \\
& -\beta) \cos \eta\left[\sum_{n=0}^{\infty} \frac{(|a|)_{n}(|b|)_{n}}{(c)_{n}(1)_{n}}-1\right] \\
& +\frac{2(1-\alpha)(1-\beta)(c-1) \cos \eta}{(|a|-1)(|b|-1)}\left[\sum_{n=0}^{\infty} \frac{(|a|-1)_{n}(|b|-1)_{n}}{(c-1)_{n}(1)_{n}}-1\right. \\
& \left.-\frac{(|a|-1)(|b|-1)}{(c-1)}\right]=2 \alpha(1-\beta) \cos \eta\left[\frac{\Gamma(c) \Gamma(c-|a|-|b|)}{\Gamma(c-|a|) \Gamma(c-|b|)}\right. \\
& -1] \\
& +\alpha(|a b|+1-c)]-[c+|a b|-|a|-|b|-\alpha(c-1)]] \leq 1 . \\
& +\beta \frac{2(1-\alpha)(1-\beta)(c-1) \cos \eta}{(|a|-1)(|b|-1)}\left[\frac{\Gamma(c-1) \Gamma(c-|a|-|b|+1)}{\Gamma(c-|a|) \Gamma(c-|b|)}\right. \\
& -\frac{2(1-\beta) \cos \eta}{(|a|-1)(|b|-1)}\left[\frac{\Gamma(c) \Gamma(c-|a|-|b|)}{\Gamma(c-|a|) \Gamma(c-|b|)}[c-|a|-|b|\right. \\
& \left.-1-\frac{(|a|-1)(|b|-1)}{(c-1)}\right]=2 \alpha(1-\beta) \cos \eta \\
& \left.\left.+\frac{\Gamma(c) \Gamma(c-|a|-|b|)}{\Gamma(c-|a|) \Gamma(c-|a|-|b|)}-|b|\right)-1\right] \\
& +(|a|-1)(|b|-1)
\end{aligned}
$$

On taking $b=\bar{a}$, an improvement of the assertion of Theorem 4 is obtained as given in Theorem 5 .

Theorem 5. Let $a \in C \backslash\{0\}$. Further let $c$ be a real number such that $c>\max \{0,2 \operatorname{Re}(a)\}, a \neq 1$. If $f \in R_{\eta}(\beta),(\beta<1)$ and the inequality

$$
\frac{2(1-\beta) \cos \eta}{(a-1)(\bar{a}-1)}\left[\frac{\Gamma(c) \Gamma(c-2 \operatorname{Re}(a))}{\Gamma(c-a) \Gamma(c-\bar{a})}[c-2 \operatorname{Re}(a)\right.
$$




$$
\begin{aligned}
& \left.+\alpha\left(|a|^{2}+1-c\right)\right]-\left[c-|a|^{2}-2 \operatorname{Re}(a)\right. \\
& -\alpha(c-1)]] \leq 1
\end{aligned}
$$

is satisfied, then $I_{a, \bar{a}, c}(f) \in S D(\alpha)$.

\section{An Integral Operator}

We now obtain results in connection with a particular integral operator $[11] G(a, b ; c ; z)$ defined by

$$
G(a, b ; c ; z)=\int_{0}^{z} F(a, b ; c ; t) d t
$$

where $F(a, b ; c ; z)={ }_{2} F_{1}(z)$ is given by $(5)$.

Theorem 6. Let $a, b \in C-\{0\}$. Also let $c$ be a real number such that $c>|a|+|b|,|a| \neq 1,|b| \neq 1$. Let $G(a, b ; c ; z)$ be given by (19). If the hypergeometric inequality

$$
\begin{aligned}
& \frac{\Gamma(c) \Gamma(c-|a|-|b|)}{\Gamma(c-|a|) \Gamma(c-|b|)}\left[\frac{c(1-\alpha)+\alpha(|a b|+1)-(|a|+|b|)}{(|a|-1)(|b|-1)}\right] \\
& -\frac{(1-\alpha)(c-1)}{(|a|-1)(|b|-1)} \leq 2
\end{aligned}
$$

is satisfied, then $G(a, b, c, z) \in S D(\alpha)$.

Proof. The function $G(a, b ; c ; z)$ has the series representation given by

$$
z+\sum_{n=2}^{\infty} \frac{(a)_{n-1}(b)_{n-1}}{(c)_{n-1}(1)_{n}} z^{n} .
$$

In view of Theorem 1 , it is enough to prove that

$$
\sum_{n=2}^{\infty}[1+\alpha(n-1)]\left|\frac{(a)_{n-1}(b)_{n-1}}{(c)_{n-1}(1)_{n}}\right| \leq 1 .
$$

Now

$$
\begin{aligned}
& \sum_{n=2}^{\infty} {[1+\alpha(n-1)]\left|\frac{(a)_{n-1}(b)_{n-1}}{(c)_{n-1}(1)_{n}}\right| \leq(1-\alpha) } \\
& \cdot \sum_{n=2}^{\infty} \frac{(|a|)_{n-1}(|b|)_{n-1}}{(c)_{n-1}(1)_{n}}+\alpha \sum_{n=2}^{\infty} \frac{n(|a|)_{n-1}(|b|)_{n-1}}{(c)_{n-1}(1)_{n}}=(1 \\
&-\alpha) \sum_{n=2}^{\infty} \frac{(|a|-1)_{n}(|b|-1)_{n}(c-1)}{(|a|-1)(|b|-1)(c-1)_{n}(1)_{n}} \\
&+\alpha \sum_{n=2}^{\infty} \frac{(|a|)_{n-1}(|b|)_{n-1}}{(c)_{n-1}(1)_{n-1}} \\
&=\frac{(1-\alpha)(c-1)}{(|a|-1)(|b|-1)}\left[\sum_{n=0}^{\infty} \frac{(|a|-1)_{n}(|b|-1)_{n}}{(c-1)_{n}(1)_{n}}-1\right. \\
&\left.-\frac{(|a|-1)(|b|-1)}{c-1}\right]+\alpha\left[\sum_{n=1}^{\infty} \frac{(|a|)_{n}(|b|)_{n}}{\left.(c)_{n}(1)_{n}\right]}\right.
\end{aligned}
$$

$$
\begin{aligned}
& =\frac{\Gamma(c) \Gamma(c-|a|-|b|)}{\Gamma(c-|a|) \Gamma(c-|b|)}\left[\frac{(1-\alpha)(c-|a|-|b|)}{(|a|-1)(|b|-1)}\right. \\
& +\alpha]-\frac{(1-\alpha)(c-1)}{(|a|-1)(|b|-1)}-1 \leq 1,
\end{aligned}
$$

by hypothesis.

A result analogous to Theorem 6 can be stated for the class $\mathrm{UCD}(\alpha)$ in Theorem 7 .

Theorem 7. Let $a, b \in C-\{0\}$. Also let $c$ be a real number such that $c>|a|+|b|+1$. Let $f \in A$ and be of the form (1). If the hypergeometric inequality

$$
\begin{aligned}
& \frac{\Gamma(c) \Gamma(c-|a|-|b|-1)}{\Gamma(c-|a|) \Gamma(c-|b|)}[c-|a|-|b|+\alpha|a b|-1] \\
& \quad \leq 2
\end{aligned}
$$

is satisfied, then $G(a, b, c, z) \in U C D(\alpha)$.

\section{Conflicts of Interest}

The authors declare that there are no conflicts of interest regarding the publication of this paper.

\section{Acknowledgments}

The author Maisarah Haji Mohd is supported by USM Short Term Grant 304/PMATHS/6313192.

\section{References}

[1] P. L. Duren, Univalent Functions, Springer, Berlin, Germany, 1983.

[2] T. Rosy, B. A. Stephen, K. G. Subramanian, and H. Silverman, "Classes of convex functions," International Journal of Mathematics and Mathematical Sciences, vol. 23, no. 12, pp. 819-825, 2000.

[3] D. Breaz, "Integral Operators on the $\mathrm{UCD}(\beta)$ class," in Proceedings of the International conference on Theory and Applications of Mathematics and informatics (ICTAMI '03, pp. 61-65, Alba Lulia, Romania, 2003.

[4] S. Sivasubramanian and J. Sokół, "Hypergeometric transforms in certain classes of analytic functions," Mathematical and Computer Modelling, vol. 54, no. 11-12, pp. 3076-3082, 2011.

[5] S. S. Varma and T. Rosy, "Certain properties of a subclass of univalent functions with finitely many fixed coefficients," Khayyam Journal of Mathematics, vol. 53, no. 1, pp. 26-33, 2017.

[6] T. Rosy, Studies on subclasses of Starlike and Convex Functions [Ph.D. thesis], University of Madras, Chennai, India, 2001.

[7] S. Ponnusamy and F. Ronning, "Duality for Hadamard products applied to certain integral transforms," Complex Variables, Theory and Application, vol. 32, no. 3, pp. 263-287, 1997.

[8] Y. E. Hohlov, "Operators and operations on the class of univalent functions," Soviet Mathematics, vol. 22, no. 10, pp. 64-69, 1978. 
[9] T. Thulasiram, K. Suchithra, T. V. Sudharsan, and G. Murugusundaramoorthy, "Some inclusion results associated with certain subclass of analytic functions inolving Hohlov operator," Revista de la Real Academia de Ciencias Exactas, Fisicas y Naturales - Serie A: Matematicas, vol. 108, no. 2, pp. 711-720, 2014.

[10] R. Nevanlinna, "Eindeutige Analytische Funktionen," in Översikt av Finska Vetenskaps-Soc. Förh, vol. 63, pp. 1-21, 6 edition, 1921.

[11] H. Silverman, "Starlike and convexity properties for hypergeometric functions," Journal of Mathematical Analysis and Applications, vol. 172, no. 2, pp. 574-581, 1993. 


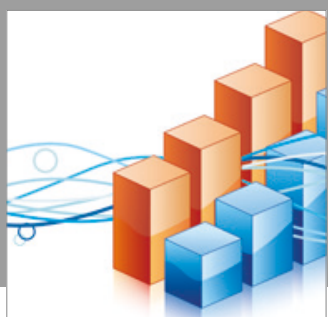

Advances in

Operations Research

vatersals

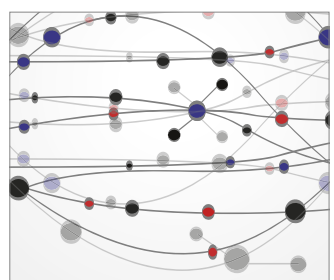

\section{The Scientific} World Journal
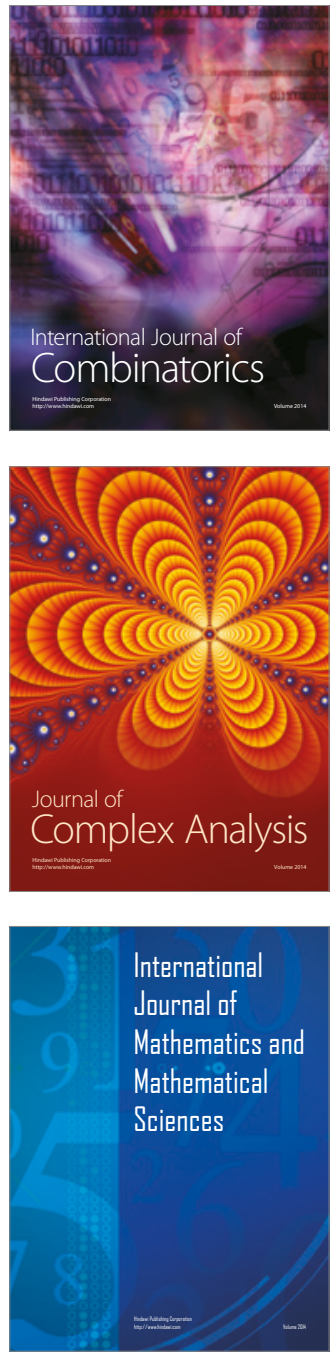
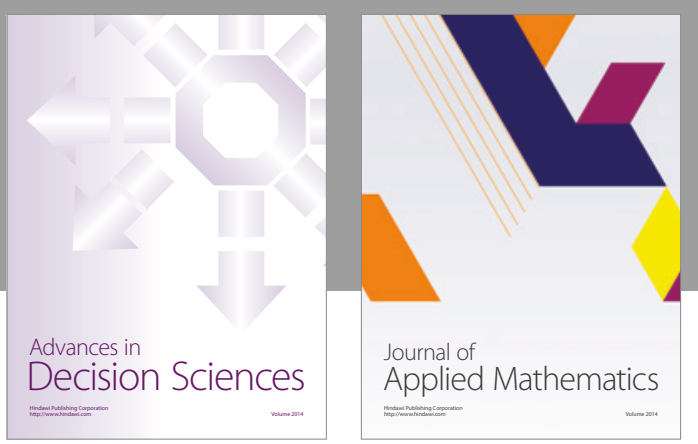

Algebra

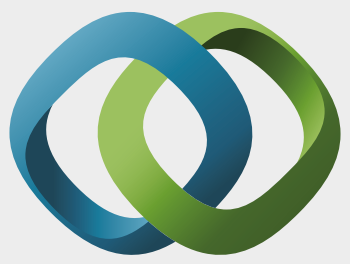

\section{Hindawi}

Submit your manuscripts at

https://www.hindawi.com
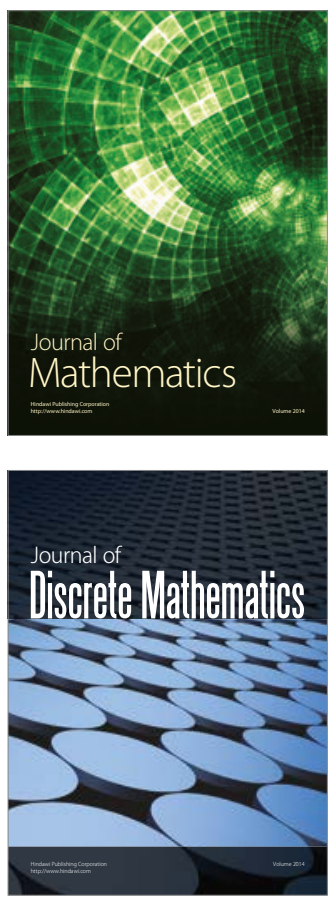

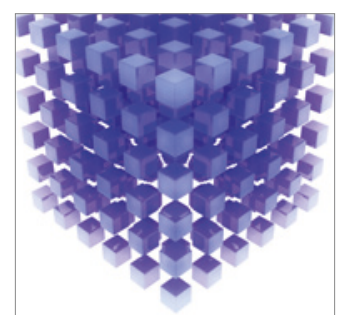

Mathematical Problems in Engineering
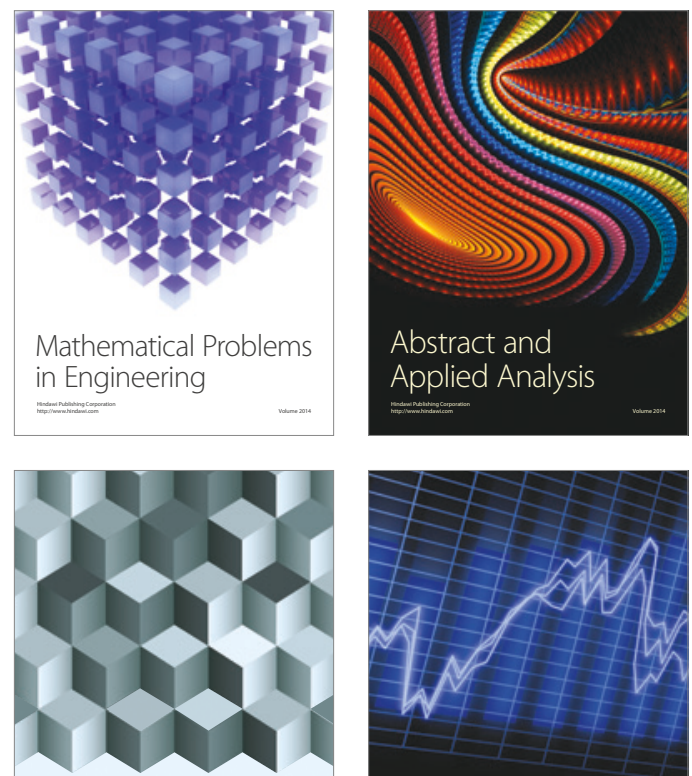

Journal of

Function Spaces

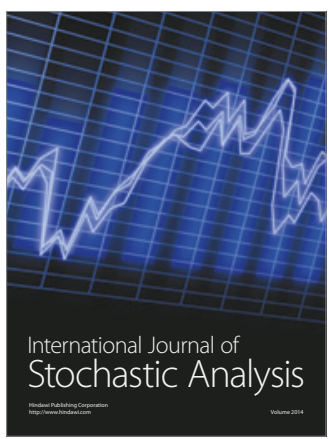

Probability and Statistics
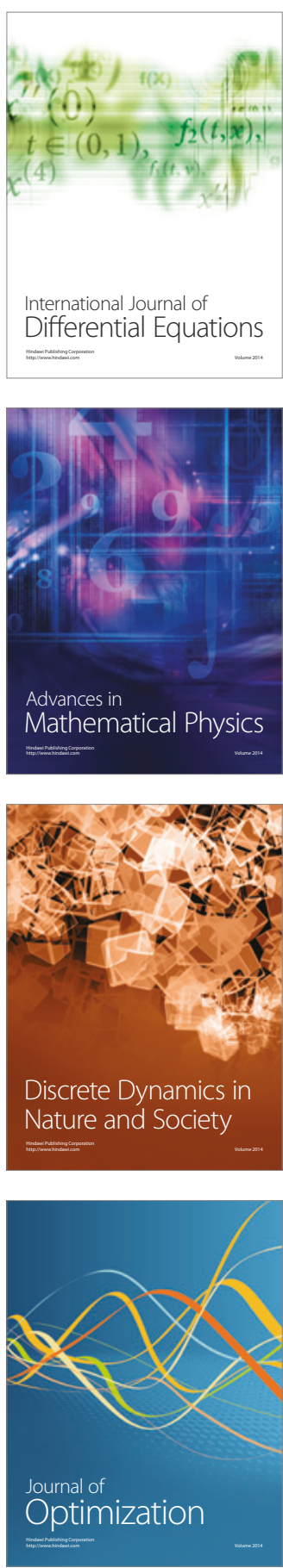